\title{
Ultrasonic imaging in air using fan-beam tomography and electrostatic transducers
}

\author{
Paul Ingleby, William M.D. Wright * \\ Department of Electrical and Electronic Engineering, University College Cork, College Road, Cork, Ireland
}

\begin{abstract}
Air-coupled ultrasonic capacitance transducers operating at frequencies of up to $1 \mathrm{MHz}$ have been employed in a fan-beam configuration for the cross-sectional tomographic imaging of temperature fields and flow fields in air, and the location of solid objects. Separate transmitter and receiver transducers were manufactured using thin polymer dielectric membranes and polished metal backplates, and used to acquire through-transmission data. The fan-beam reconstruction was developed in LabVIEW ${ }^{\circledR}$ using a re-bin routine combined with a filtered backprojection algorithm and a difference technique to generate the cross-sectional images. The system was first used to reconstruct images showing the locations of solid objects positioned within the scanned region through interpretation of the arrival time of the transmitted ultrasound. The technique was then extended to image the temperature fields produced in air above a small heat source and the flow field produced by a nozzle connected to a regulated compressed air source. Reconstructed temperatures were within $4 \%$ of the measured background air temperature and $9 \%$ of the air temperature measured above the heat source. Reconstructed images of the flow field above a small nozzle were also presented, showing that the horizontal component of the flow velocity could be resolved using this method. (C) 2002 Elsevier Science B.V. All rights reserved.
\end{abstract}

Keywords: Air-coupled ultrasound; Fan-beam tomography; Capacitance transducer

\section{Introduction}

Tomographic reconstruction [1] is a well-established technique, by which cross-sectional images of spatial variations in ultrasonic properties may be formed. The region to be imaged is usually interrogated with ultrasound from a range of angles and positions at the periphery of the plane of interest, and an image produced using changes in a physical variable of the transmitted signals such as propagation time or amplitude. There are two main techniques for this type of tomography. Series expansion techniques [2] employ iterative methods but are computationally inefficient. Fourier transform methods, such as the filtered backprojection theorem [3], are more efficient but require a well-defined, regular sampling geometry in either a parallel-beam or fan-beam configuration. Tomographic imaging in air has been limited primarily to the location of solid objects $[4,5]$, and this lack of application has been due in

\footnotetext{
${ }^{*}$ Corresponding author. Tel.: +353-21-4902213; fax: +353-214271698.

E-mail address: bill.wright@ucc.ie (W.M.D. Wright).
}

no small part to the difficulty of producing ultrasonic transducers that operate effectively in air and other gases. Transducers based on an electrostatic or capacitance principle have generated much recent interest due to their ability to efficiently generate and detect broadband ultrasound in air at $\mathrm{MHz}$ frequencies, and there is a considerable body of research into the fabrication, characterisation and modelling of such devices (e.g. [69]). In previous work by Wright et al. [10], ultrasonic tomographic reconstruction of temperature and flow fields was achieved in air using air-coupled capacitance transducers and a parallel-beam geometry. In the present study, this technique is extended to a fan-beam geometry. This configuration was used initially for the location and interrogation of solid objects placed within the scan area. It was then used to reconstruct temperature and flow fields generated in air above a small heat source and air jet, respectively. This technique could potentially be used to remotely characterise plumes and sources of air pollution. The reconstruction software was written in LabVIEW $^{\circledR}$ using a re-bin routine that extracted parallel-beams from the fan-beam geometry data [11]. These projections were then scaled and interpolated 
so that a parallel-beam filtered backprojection algorithm could be employed for the image reconstruction.

\section{Sampling geometry}

A typical fan-beam sampling geometry employed is shown schematically in Fig. 1. A source transducer $x$ is positioned on the perimeter of a circular area of interest and a receiver $y$ is scanned through a number of regularly spaced positions opposite the source to produce a fanshaped series of ultrasonic rays known as a projection. This procedure is then repeated at different source positions $x^{\prime}$ until a series of projections is obtained, from which an image can be reconstructed. Certain rays in different projections will be parallel to each other, e.g. rays $r_{1}$ and $r_{2}$ in Fig. 1, and a re-bin routine may then be employed to extract sets of parallel rays from the fanbeam data. These parallel rays will initially be nonuniformly spaced in $s$ and of different lengths. Therefore an interpolation routine must be employed, using a reference velocity that is obtained from the propagation time of the first ray. It is assumed that as this first ray path is at the edge of the scanned area and away from any disturbances, the ultrasonic velocity along its path is also the background velocity throughout the scanned region. The interpolated data is then reconstructed using a filtered backprojection algorithm, the details of which have been discussed in earlier studies [1,3]. For any ray path $x y$, both the local speed of sound and the gas flow velocity will have an effect on the propagation time $t_{x y}$, given by

$t_{x y}=\int_{r_{x}}^{r_{y}} \frac{\mathrm{d} r}{c_{r}+\underline{\mathbf{v}} \cdot \hat{\mathbf{r}}}$

where $c_{r}$ is the speed of sound at any position $r$ along the ray path $x y, \underline{\mathbf{v}}$ is the local flow velocity of the gas and $\hat{\mathbf{r}}$ is a unit vector parallel with the ray path and pointing in the direction of integration. As the local speed of sound is also related to temperature by the well-known relation [12]:

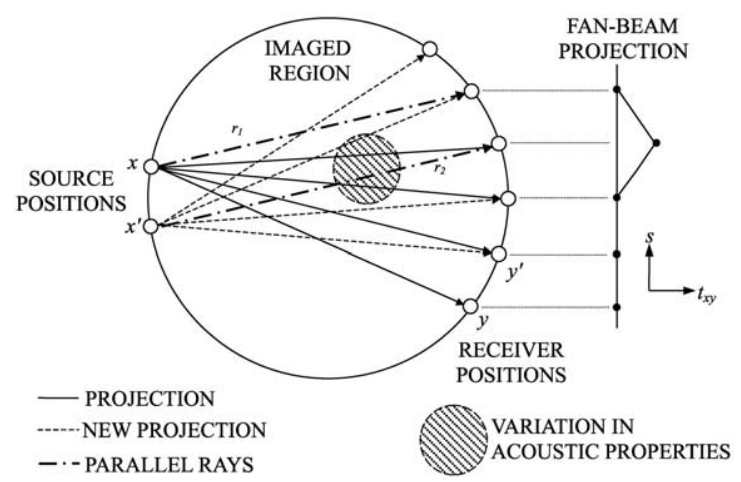

Fig. 1. Schematic diagram showing the sampling geometry for fanbeam tomography. $c_{r}=331.31 \sqrt{\frac{T}{273.16}}$

where $T$ is the air temperature in Kelvin, a reconstructed image of the spatial variation in slowness $\left(c_{r}+\underline{\mathbf{v}} \cdot \hat{\mathbf{r}}\right)^{-1}$ within the scanned area will indicate the presence of solid objects, gas flows, or changes in temperature. Further details of this temperature and flow reconstruction technique can be found in earlier work by Wright et al. [10].

\section{Experimental setup}

The fan-beam scan geometry shown in Fig. 1 demonstrates that, in order to maximise the number of rays in each projection, a divergent source and receiver are required. A pair of electrostatic devices, consisting of polished convex metal backplates with a $10 \mathrm{~mm}$ radius of curvature and $5 \mu \mathrm{m}$ thick metallised PET dielectric film, was employed for both generation and detection of ultrasound. These devices operated at a frequency of 400 $\mathrm{kHz}$ with a $-6 \mathrm{~dB}$ bandwidth of $300 \mathrm{kHz}$, and their basic operation has been detailed elsewhere [6-9].

The experimental configuration is shown schematically in Fig. 2. The source transducer was biased using a 100 V DC supply and driven by a Panametrics pulserreceiver (model 500PR) which delivered a negative spike of up to $-250 \mathrm{~V}$ with a pulse energy of up to $19.4 \mu \mathrm{J}$. The receiver was connected to a Cooknell CA6/C charge amplifier, with a sensitivity of $250 \mathrm{mV} \mathrm{pC}^{-1}$, and SU2/C power supply providing a DC bias of $100 \mathrm{~V}$. The transducers were mounted on Daedal rotary stages driven using stepper motors and custom electronics under RS232 control to an accuracy of $\pm 0.02^{\circ}$. The received waveforms were captured on a Tektronix TDS224 digital oscilloscope and transferred to a PC via an RS232 interface for storage and analysis.

Each scan consisted of 37 receiver positions at $5^{\circ}$ intervals opposite the source, and 72 source locations in $360^{\circ}$. Not all of this data was required for the re-bin routine to operate successfully, but that redundancy in

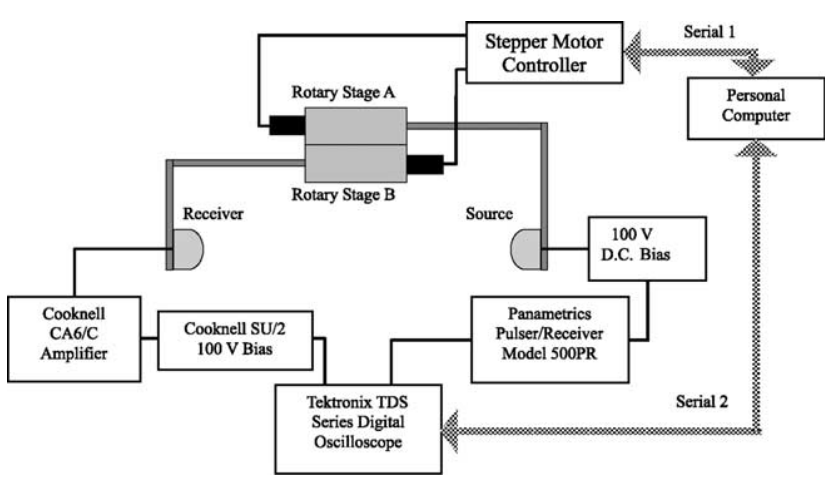

Fig. 2. The experimental apparatus employed for fan-beam reconstructions using convex air-coupled transducers. 
the acquisition was necessary to ensure that all of the required parallel ray paths were collected. Due to the volume of data required (2664 waveforms), the data acquisition process and transducer positioning were automated, allowing computer control via an RS232 interface using routines written in LabVIEW ${ }^{\circledR}$. Each waveform was acquired in approximately $10 \mathrm{~s}$, giving a total scan time of about $8 \mathrm{~h}$. This data acquisition time could be reduced further, by using a faster interface, transducer arrays, and multiplexing the sources and receivers.

\section{Results and discussion}

\subsection{Solid objects}

Fig. 3 shows the reconstructed image of acoustic velocity (reciprocal of slowness) in the scan area with a 21 $\mathrm{mm}$ diameter Nylon cylinder located at the coordinates $(5,-5) \mathrm{mm}$. The reconstructed background velocity in air was $350 \mathrm{~m} \mathrm{~s}^{-1}$, which is within $1 \%$ of the speed of sound at room temperature $\left(347 \mathrm{~m} \mathrm{~s}^{-1}\right)$. The speed of sound within the cylinder was reconstructed to be 340 $\mathrm{m} \mathrm{s}^{-1}$, yet should be $2400 \mathrm{~m} \mathrm{~s}^{-1}$. As no waveform could be measured travelling directly through the cylinder, the reconstructed speed of sound within the solid is due to diffraction of the waves in air around its edge. The larger propagation time associated with these longer ray paths makes the velocity within the cylinder appear lower than the velocity in air. However, the reconstruction still extracted the true position and diameter of the cylinder, shown by the broken white line.

Fig. 4 shows the reconstructed image with the centre of a $25 \mathrm{~mm}$ square aluminium block located at the coordinates $(0,20) \mathrm{mm}$. A threshold amplitude was employed on the sampled data, as there were no propagation times associated with occluded rays, which in-

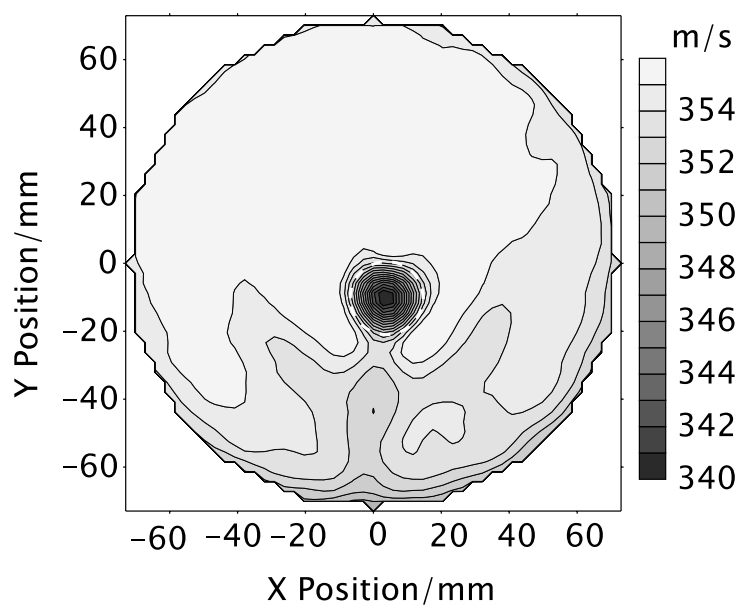

Fig. 3. Tomographic image with a $21 \mathrm{~mm}$ diameter Nylon cylinder in the scan area.

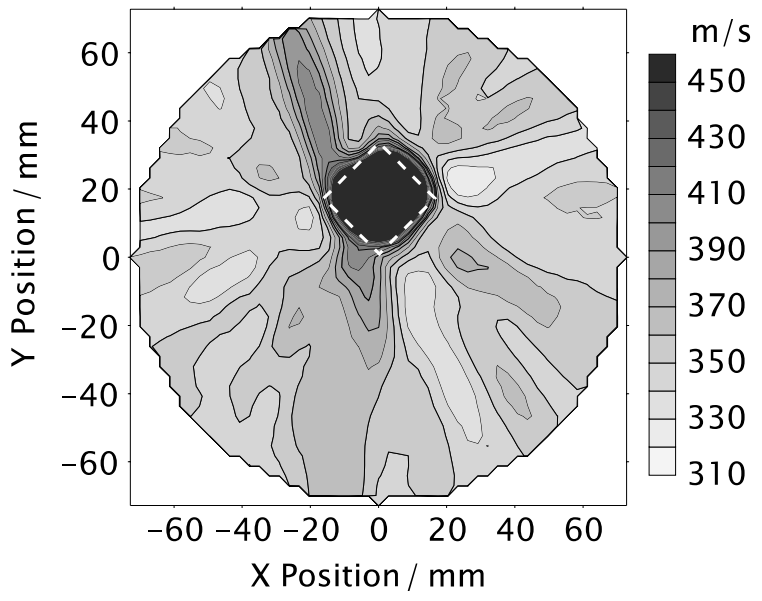

Fig. 4. Tomographic image with a $25 \mathrm{~mm}$ square aluminium bar in the scan area.

troduced noise into the reconstruction. For this reason the speed of sound within the object is entirely artificial and is determined by the propagation time delay inserted to replace the occluded data. A reconstruction with multiple objects within the scan area was also attempted using two $21 \mathrm{~mm}$ diameter cylinders positioned at the coordinates $(20,-10)$ and $(-30,-10)$. It can be seen from Fig. 5 that the software was able to locate the two objects and to reconstruct the background velocity, although the shapes of the objects have been distorted as one object occludes the other in several rays in the scan. As the use of artificial data generated by the amplitude threshold routine increases, the quality of the image is adversely effected.

\subsection{Temperature and flow fields}

In order to image the spatial distribution of temperature within the scan area, a $6 \mathrm{~mm}$ diameter soldering

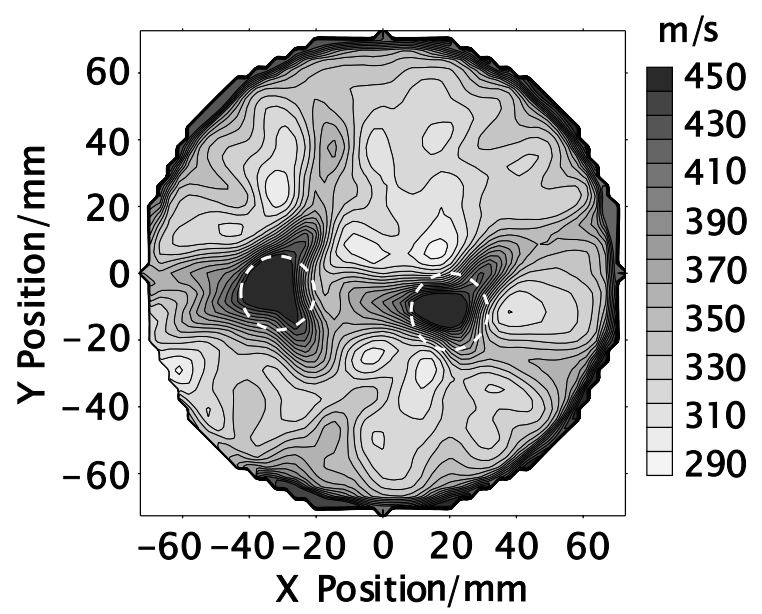

Fig. 5. Tomographic image with two $21 \mathrm{~mm}$ solid cylinders in the scan area. 
iron at a constant temperature of $573 \mathrm{~K}$ was held centrally below the scanning plane. Reconstructed slowness values were converted into spatial variations in temperature using Eq. (2) and are shown in Fig. 6, and a definite heat plume can be seen in the centre of the scan. The background air temperature extracted from the tomography was between 306 and $314 \mathrm{~K}$, and compared to the actual air temperature of $294 \mathrm{~K}$ measured using a $1 \mathrm{~mm}$ diameter $\mathrm{K}$-type thermocouple, gave an error in the reconstruction of between $4 \%$ and $7 \%$. The temperature directly above the heat source was reconstructed to be $330 \mathrm{~K}$, and was measured as $362 \mathrm{~K}$, which gave an error of $9 \%$. The physical size of the transducers meant that considerable spatial averaging occurred to the signals passing through the scan area, which would affect the accuracy of the reconstruction. The background air temperature also fluctuated by $\pm 5 \mathrm{~K}$ during the data collection, which means that the temperatures would be time averaged. As a difference technique based on the first ray of the first projection was used, any variations in ambient temperature after this initial acquisition would affect the reconstruction. It is also likely that insufficient ray paths were used to achieve the required spatial resolution.

Fig. 7 shows a tomographic reconstruction of the temperature field in air above two heat sources, at temperatures of 573 and $473 \mathrm{~K}$, located below the scanning plane at coordinates $(10,-20)$ and $(-15,30)$ respectively. The image indicates a background temperature of between 305 and $310 \mathrm{~K}$ and shows the two heat plumes to be at 325 and $319 \mathrm{~K}$. The higher temperature plume can clearly be distinguished in this image, however the shape and position of the other plume was obscured by background noise.

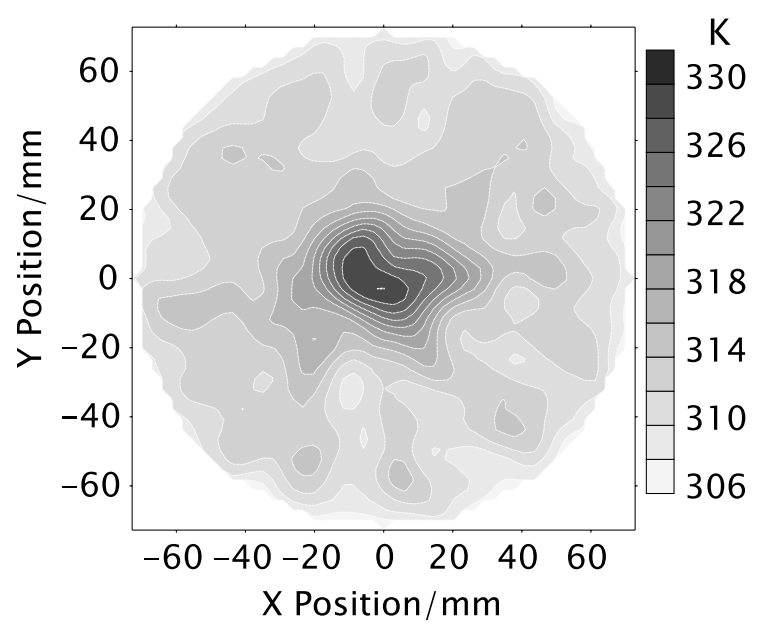

Fig. 6. Tomographic image of the temperature field in air above a 573 $\mathrm{K}$ heat source.

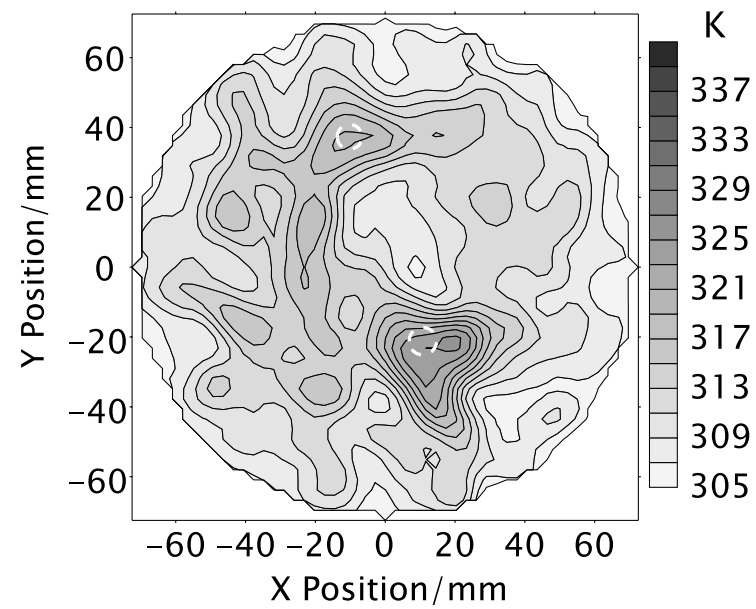

Fig. 7. Tomographic image of the temperature field above two heat sources at 574 and $473 \mathrm{~K}$ positioned below the scanning plane.

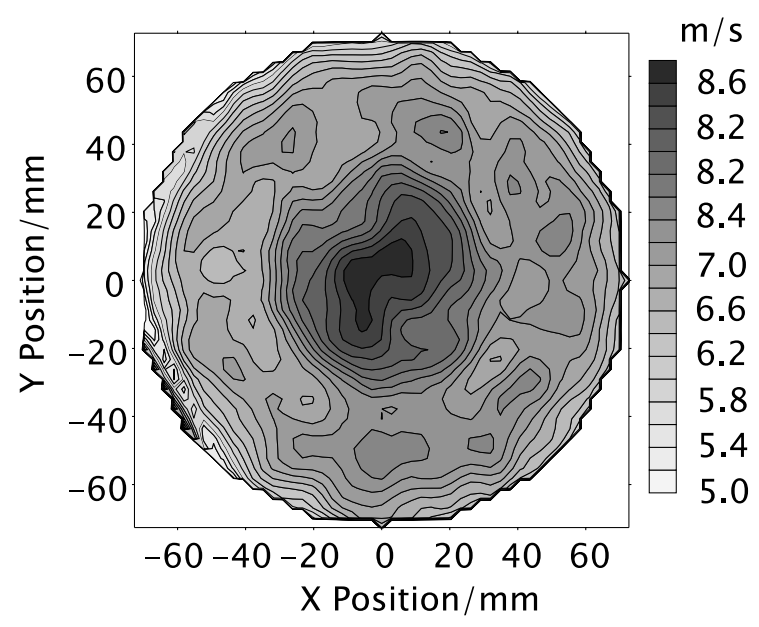

Fig. 8. Tomographic reconstruction of the horizontal flow component image above a vertical air jet.

Finally, Fig. 8 shows a reconstruction of the flow profile above a $2.7 \mathrm{~mm}$ diameter nozzle connected to a compressed air source with a flow rate of $0.51 \mathrm{~s}^{-1}$ measured using a Platon A10HS flowmeter. This figure shows the horizontal components of the flow velocity of the air jet, reconstructed from slowness data using a value of $347 \mathrm{~m} \mathrm{~s}^{-1}$ for $c_{r}$ in Eq. (1). The maximum horizontal component of the flow is shown to be 8.6 $\mathrm{m} \mathrm{s}^{-1}$ at the centre of the scan area, which is in good agreement with theory outlined in previous work [10], which predicts a maximum horizontal flow velocity of between 8.0 and $9.4 \mathrm{~m} \mathrm{~s}^{-1}$ at a height of $45 \mathrm{~mm}$ above the nozzle. However the information reconstructed within the scan area is limited by the same constraints concerning transducer size, turbulence, time averaging, compressibility and ray bending. 


\section{Conclusions}

Fan-beam tomography has been employed for the tomographic imaging of temperature and flow fields in air, and the location of solid objects, using a pair of convex electrostatic transducers. A re-bin routine that extracted parallel ray paths from a fan-beam configuration was combined with a filtered backprojection algorithm to form images of the spatial variation in slowness. This process was successful in determining the position and size of solid objects within the scan area and was then extended to imaging temperature and flow fields. Temperature reconstructions were shown to give an accuracy of within $4 \%$ of the background temperature and $9 \%$ of the temperature above a heat source when compared to data gathered using a commercial thermocouple. Reconstructed images of the flow field above a small nozzle were also presented, showing that the horizontal component of a flow velocity can be resolved using this method.

\section{Acknowledgements}

This work was supported by the European Commission through the Marie Curie Individual Fellowship Scheme (contract number HPMF-CT-1999-00038). The authors would also like to thank Prof. D.A. Hutchins of Warwick University for discussions during the development of the software.

\section{References}

[1] A.C. Kak, M. Slaney, Principles of Computerized Tomographic Imaging, IEEE Press, New York, 1988.

[2] Y. Censor, Finite series-expansion reconstruction methods, Proc. IEEE 71 (1983) 409.

[3] A.J. Devaney, Fast filtered backpropagation algorithm for ultrasound tomography, IEEE Trans. UFFC 34 (1987) 330.

[4] G.J. Brown, D. Reilly, Ultrasonic tomographic imaging of solid objects in air using an array of fan-shaped-beam electrostatic transducers, Ultrasonics 34 (1996) 111.

[5] G.J. Brown, D. Reilly, D. Mills, Development of an ultrasonic tomography system for application in pneumatic conveying, Meas. Sci. Technol. 7 (1996) 396.

[6] H. Carr, C. Wykes, Diagnostic measurements in capacitive transducers, Ultrasonics 31 (1993) 13.

[7] D. Schindel, D. Hutchins, L. Zou, M. Sayer, The design and characterization of micromachined air-coupled capacitance transducers, IEEE Trans. UFFC 42 (1995) 42.

[8] M. Oksanen, J. Varis, J. Hietanen, J. Wu, Quantitative theory for $\mathrm{V}$-groove capacitive transmitting transducers, Ultrasonics 35 (1997) 205.

[9] B. Khuri-Yakub, F. Degertekin, X. Jin, S. Calmes, I. Ladabaum, S. Hansen, X. Zhang, Silicon micromachined ultrasonic transducers, Proc. IEEE Ultrason. Symp. 2 (1998) 985.

[10] W.M. Wright, D.W. Schindel, D.A. Hutchins, P.W. Carpenter, D.P. Jansen, Ultrasonic tomographic imaging of temperature and flow fields in gases using air-coupled capacitance transducers, J. Acoust. Soc. Am. 104 (1998) 3446.

[11] T.M. Peters, R.M. Lewitt, Computed tomography with fan-beam geometry, J. Comput. Assist. Tomog. 1 (1977) 429.

[12] R. Hickling, S.P. Marin, The use of ultrasound for gauging and proximity sensing in air, J. Acoust. Soc. Am. 79 (1986) 1151. 